\title{
Resting-State Functional Connectivity and Cognition After Major Cardiac Surgery in Older Adults without Preoperative Cognitive Impairment: Preliminary Findings
}

\author{
Jeffrey N. Browndyke, PhD, ** Miles Berger, MD, PhD, ${ }^{\S}$ Todd B. Harshbarger, PhD, ${ }^{\neq}$ \\ Patrick J. Smith, PhD, * William White, MPH, ${ }^{\dagger \dagger}$ Tiffany L. Bisanar, RN, ${ }^{\dagger \dagger}$ \\ John H. Alexander, MD, MHS, ${ }^{*}$ Jeffrey G. Gaca, MD, ${ }^{\mathcal{S}}$ Kathleen Welsh-Bohmer, PhD,* \\ Mark F. Newman, MD, ${ }^{+\dagger}$ and Joseph P. Mathew, MD, MHS, MBA ${ }^{+\dagger}$
}

OBJECTIVES: To look for changes in intrinsic functional brain connectivity associated with postoperative changes in cognition, a common complication in seniors undergoing major surgery, using resting-state functional magnetic resonance imaging.

DESIGN: Objective cognitive testing and functional brain imaging were prospectively performed at preoperative baseline and 6 weeks after surgery and at the same time intervals in nonsurgical controls.

SETTING: Academic medical center.

PARTICIPANTS: Older adults undergoing cardiac surgery $(\mathrm{n}=12)$ and nonsurgical older adult controls with a history of coronary artery disease $(\mathrm{n}=12)$; no participants had cognitive impairment at preoperative baseline (MiniMental State Examination score $>27$ ).

MEASUREMENTS: Differences in resting-state functional connectivity (RSFC) and global cognitive change relationships were assessed using a voxel-wise intrinsic connectivity method, controlling for demographic factors and pre- and perioperative cerebral white matter disease volume. Analyses were corrected for multiple comparisons (false discovery rate $P<.01$ ).

RESULTS: Global cognitive change after cardiac surgery was significantly associated with intrinsic RSFC changes in

From the *Geriatric Behavioral Health Division, Department of Psychiatry and Behavioral Sciences, Duke University Medical Center; ${ }^{\dagger}$ Institute for Brain Sciences; ${ }^{*}$ Brain Imaging and Analysis Center, Duke University;

${ }^{\S}$ Division of Neuroanesthesiology, Department of Anesthesiology;

"Department of Radiology; " Behavioral Medicine Division, Department

of Psychiatry and Behavioral Sciences; ${ }^{\dagger \dagger}$ Department of Anesthesiology;

${ }^{\sharp}$ Division of Cardiology, Department of Medicine; and ${ }^{\S}$ Division of

Cardiothoracic Surgery, Department of Surgery, Duke University Medical

Center, Durham, North Carolina.

Address correspondence to Jeffrey N. Browndyke, Geriatric Behavioral Health Division, Department of Psychiatry and Behavioral Sciences, Duke University Medical Center, 2200 West Main Street, Suite A-200, Durham, NC 27705. E-mail: j.browndyke@duke.edu

DOI: $10.1111 /$ jgs. 14534 regions of the posterior cingulate cortex and right superior frontal gyrus - anatomical and functional locations of the brain's default mode network (DMN). No statistically significant relationships were found between global cognitive change and RSFC change in nonsurgical controls.

CONCLUSION: Clinicians have long known that some older adults develop postoperative cognitive dysfunction (POCD) after anesthesia and surgery, yet the neurobiological correlates of POCD are not well defined. The current results suggest that altered RSFC in specific DMN regions is positively correlated with global cognitive change 6 weeks after cardiac surgery, suggesting that DMN activity and connectivity could be important diagnostic markers of POCD or intervention targets for potential POCD treatment efforts. J Am Geriatr Soc 2016.

Key words: cardiac surgical procedures; anesthesia; brain; cognition; functional neuroimaging

$\mathbf{P}$ ostoperative cognitive dysfunction (POCD) has been described in individuals undergoing cardiac and noncardiac surgery and occurs more often in individuals aged 60 and older, ${ }^{1}$ but there is no consensus on exactly how to measure POCD, when after surgery to measure it, or how much a person's cognitive performance must decline to be considered clinically relevant. Human clinical and animal studies have suggested that POCD may result from unresolved neuroinflammation, ${ }^{2}$ Alzheimer's disease-associated pathology ${ }^{3}$ specific anesthetics, ${ }^{4}$ perioperative cerebrovascular damage, ${ }^{5}$ or an effect of preexisting health conditions such as metabolic syndrome, ${ }^{6}$ but the relative contribution of these possible factors to human POCD is unclear. Furthermore, whether structural or functional deficits in specific brain regions or global dysfunction underlie human POCD is unknown. 
Resting-state functional connectivity (RSFC) is a reliable neurophysiological phenomenon characterized by shared coherent, spontaneous, low-frequency blood oxygen level-dependent (BOLD) signal fluctuations among functionally related brain regions in the absence of task-related activation. $^{7}$ RSFC networks have been identified for the motor system; task-negative, introspective thought and memory; stimulus salience; sensorimotor integration; auditory processing; and higher-order executive functioning. The most-studied RSFC network is the default mode network (DMN), a group of functionally connected posterior and anterior cortical regions that are active when individuals are at rest or internally focused and that become less active when they shift their focus and cognitive effort to external tasks. ${ }^{8}$ Regions critical to the DMN, such as the posterior cingulate cortex (PCC), have been consistently identified as being densely anatomically interconnected with the rest of the brain and play a central role in global cerebral communication processes. ${ }^{9}$ RSFC alterations in DMN regions have been found in several conditions associated with cognitive decline or dysfunction, including advanced aging, ${ }^{10}$ Alzheimer's disease, ${ }^{11}$ and delirium. ${ }^{12}$ It was hypothesized that perioperative changes in DMN RSFC would be correlated with postoperative cognitive changes in individuals undergoing cardiac surgery but not in nonsurgical controls.

\section{METHODS}

The Duke University Medical Center Institutional Review Board approved this study, and all enrolled study participants provided written informed consent.

\section{Participants}

Individuals aged 60 and older scheduled to undergo coronary artery bypass grafting (CABG) or valve replacement (VR) surgery with cardiopulmonary bypass were prospectively enrolled. Nonsurgical controls with coronary artery disease (CAD), as evidenced by a prior myocardial infarction or evidence of $\mathrm{CAD}$ on cardiac catheterization, were recruited from a cardiology clinic. Nonsurgical controls could not be under consideration for surgical revascularization within 6 weeks of the baseline study visit. Individuals with a history of cortical stroke, alcoholism, psychiatric illness, renal failure, less than a seventh-grade education, nonnative English speaking, or a baseline Mini Mental Status Examination (MMSE) score of 26 or less, or those who were unsafe for $3 \mathrm{~T}$ magnetic resonance imaging (MRI) were excluded (Table 1, Appendix S1).

\section{Perioperative Management}

In participants undergoing surgery, anesthesia was induced and maintained with midazolam, fentanyl, and isoflurane or sevoflurane. All participants undergoing surgery underwent nonpulsatile hypothermic $\left(30-32^{\circ} \mathrm{C}\right)$ cardiopulmonary bypass $(\mathrm{CPB})$ with a membrane oxygenator and an arterial line filter. The pump was primed with crystalloid, and serial hematocrit levels were kept at 0.21 or greater. Before initiation of $\mathrm{CPB}$, all participants undergoing surgery received heparin anticoagulation (300$400 \mathrm{U} / \mathrm{kg}$ ) to achieve a target activated coagulation time of greater than 480 seconds. Perfusion was maintained at pump flow rates of 2 to $2.4 \mathrm{~L} / \mathrm{min}$ per $\mathrm{m}^{2}$ throughout $\mathrm{CPB}$ to maintain mean arterial pressure at 50 to $80 \mathrm{mmHg}$. See Table 1 for surgical variable data.

\section{Neurocognitive Procedures}

Participants were administered standardized neuropsychological assessment measures designed to assess auditory-verbal learning, immediate and delayed memory recall, visual immediate and delayed memory recall, complex attention, visuomotor performance and processing speed, manual dexterity, and complex executive functioning skills (Table 2, Appendix S1). All tests were administered at preoperative baseline $(0.70 \pm 0.38$ weeks before surgery) and approximately 6 weeks postoperatively $(6.56 \pm 1.96$ weeks). There were no participant losses, and cognitive testing was completed for all participants at baseline and follow-up.

Participants undergoing surgery were screened for agitation and sedation and postoperative delirium on postoperative Days 1 to 3 . Although sedation and partial delirium symptoms were a factor in four of 12 participants undergoing surgery during the postoperative period, none met full Confusion Assessment Method ${ }^{13}$ delirium diagnostic criteria.

\section{Neuroimaging Procedures and Data Acquisition}

Standard anatomical images and functional data were acquired on a 3T MRI scanner (MR750, General Electric Co., Fairfield, CT) with an eight-channel head coil. Anatomical data consisted of high-resolution T1weighted fast spoiled gradient-echo oblique axial acquisition $(256 \times 256$ matrix, 256-mm field-of-view (FOV), $11^{\circ}$ flip angle, 136 1-mm-thick slices, echo time (TE) $3.0 \mathrm{~ms}$, repetition time (TR) $6.93 \mathrm{~ms}$ ) and $\mathrm{T} 2$ fluid attenuation inversion recovery (FLAIR) oblique axial acquisition $\left(128 \times 128\right.$ matrix, $256-\mathrm{mm}$ FOV, $90^{\circ}$ flip angle, 68 2-mm-thick slices, TE $145.6 \mathrm{ms,} \mathrm{TR}$ $11,000 \mathrm{~ms}$, inversion time (TI) 2,250 ms) scans. Restingstate functional MRI (rs-fMRI) data were acquired using a sensitivity-encoding, spiral-in, oblique, axial, slice-interleaved acquisition $\left(64 \times 64\right.$ matrix, $256-\mathrm{mm}$ FOV, $60^{\circ}$ flip angle, 34 4-mm-thick slices, TE $30 \mathrm{~ms}$, TR 3,000 ms, sensitivity encoding factor 2). Eighteen seconds was discarded from the beginning of the rs-fMRI sequence to correct for initial MR signal fluctuation, after which 124 time points (6.2 minutes) of data were retained for RSFC analysis. During the rs-fMRI data collection, all subjects were instructed to look at a black crosshair centered on a white background. Physiological (respiration, heart rate) and movement data were collected during rs-fMRI scanning for later RSFC data signal correction methods. Physiological data were detected using a respiration stretch transducer and finger pulseoximeter connected to amplifiers (Biopac Systems, Inc., Goleta, CA) sampling at $100 \mathrm{~Hz}$ and synchronized to the trigger pulse associated with the first MR image acquisition using CIGAL software (https://www.nitrc.org/ projects/cigal/ $)^{14}$ (Appendix S1). 
Table 1. Demographic, General Health, and Surgical Characteristics

\begin{tabular}{|c|c|c|c|c|}
\hline Characteristic & Surgical, $n=12$ & Controls, $n=12$ & Statistic $^{a}$ & $P$-Value \\
\hline \multicolumn{5}{|l|}{ Demographic } \\
\hline Age, mean $\pm S D$ & $69.7 \pm 7.3$ & $70.4 \pm 7.9$ & -0.24 & .82 \\
\hline Male & 8 & 11 & 2.27 & .32 \\
\hline Female & 4 & 1 & & \\
\hline Weight, $\mathrm{kg}$, mean $\pm \mathrm{SD}$ & $76.9 \pm 17.4$ & $86.4 \pm 10.7$ & -1.61 & .12 \\
\hline White & 11 & 12 & 1.04 & $>.99$ \\
\hline Asian & 1 & 0 & & \\
\hline \multicolumn{5}{|l|}{ Health } \\
\hline Diabetes mellitus, $\mathrm{n}$ & 3 & 5 & 0.75 & .67 \\
\hline History of hypertension, $n$ & 6 & 11 & 3.23 & .07 \\
\hline Previous myocardial infarction, $\mathrm{n}$ & 1 & 1 & 0.00 & $>.99$ \\
\hline 0 & 7 & & & \\
\hline 1 & 0 & & & \\
\hline 2 & 0 & & & \\
\hline 3 & 1 & & & \\
\hline 4 & 1 & & & \\
\hline \multicolumn{5}{|l|}{ Surgical procedure, $n$} \\
\hline Valve & 10 & & & \\
\hline CABG & 1 & & & \\
\hline CABG plus valve replacement & 1 & & & \\
\hline Left ventricular ejection fraction, mean $\pm S D$ & $54.2 \pm 5.1$ & & & \\
\hline Mean pump cardiopulmonary bypass time, minutes, mean \pm SD & $145.9 \pm 65.6$ & & & \\
\hline Mean cross clamp time, minutes, mean \pm SD & $83.7 \pm 35.3$ & & & \\
\hline
\end{tabular}

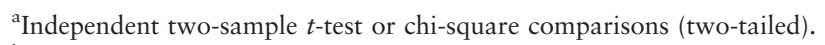

${ }^{\mathrm{b}}$ Ratio of white matter hyperintensity volume to total intracranial volume.

$\mathrm{SD}=$ standard deviation; $\mathrm{CABG}=$ coronary artery bypass graft.

\section{Neuroimaging Data Preprocessing}

Neuroimaging data were spatially preprocessed and analyzed using Statistical Parametric Mapping version 8 (SPM8, Wellcome Institute, London, UK) in MATLAB (MathWorks, Inc., Natick, MA). SPM default gray and white matter and cerebrospinal fluid anatomical segmentation parameters were applied to the T1 anatomical segmented and coregistered FLAIR and functional data for nonlinear alignment with Montreal Neurologic Institute atlas space $\left(2 \mathrm{~mm}^{3}\right.$ isotropic voxels). Functional data were spatially smoothed using a Gaussian 8 -mm full width at half maximum filter. Coregistered and normalized FLAIR data were interrogated for ischemic white matter lesion volumes at each time point using the MATLAB Lesion Segmentation Tool toolbox for SPM8.

First-level covariate correction for each participants' rs-fMRI data included composite motion greater than $0.5 \mathrm{~mm}$, physiological signal (respiration and heart rate), and BOLD scan-to-scan signal artifact greater than $3.0 \mathrm{z}$ of global mean signal. Linear regression of confounding effects was conducted using artifact detection tools (NITRC; https://www.nitrc.org/projects/artifact_detect/) and A component-based noise correction method ${ }^{15}$ to maintain temporal resolution of processed data and to avoid the induction of anticorrelations or potential default-mode network signal loss associated with global signal regression. Functional data were band-pass frequency filtered $(0.008-0.09 \mathrm{~Hz})$, and session-specific temporal linear detrending occurred after confound removal regression.

\section{Analyses}

\section{Demographic Variables and Cognitive Outcomes}

Independent two-sample $t$-tests or chi-square test comparisons (where appropriate) were conducted for all demographic variable comparisons and to assess differences between groups in cognitive outcomes (two-tailed $P<.05$; Table 1). Planned cognitive baseline and outcome comparisons involved raw scores at baseline and comparison of computed reliable change index (RCI) for each variable. RCI values, expressed as z-scores, reflect the magnitude of perioperative cognitive change, controlling for expected mean performance change, practice effects, and normal test-retest reliability observed in the nonsurgical controls (Appendix S1). ${ }^{16}$ Mean total RCI, calculated as the mean of all the RCI scores for each of the 12 cognitive variables (Table 2), was used as an aggregate measure of global perioperative cognitive change. Five of the 12 participants undergoing surgery had RCI values less than -1.64 on two or more test variables, which some groups have used as a criterion for dichotomous determination of POCD. ${ }^{17}$ 
Table 2. Cognition at Presurgical Baseline and Postoperative Change at 6-Week Follow-Up

\begin{tabular}{|c|c|c|c|c|c|c|c|c|}
\hline \multirow[b]{3}{*}{ Cognitive Tests } & \multicolumn{3}{|c|}{ Baseline Performance } & \multicolumn{5}{|c|}{ Postoperative Cognitive Change ${ }^{a}$} \\
\hline & $\begin{array}{l}\text { Surgical, } \\
n=12\end{array}$ & $\begin{array}{c}\text { Controls, } \\
n=12\end{array}$ & \multirow{2}{*}{$P$-Value } & $\begin{array}{l}\text { Surgical, } \\
\mathrm{n}=12\end{array}$ & $\begin{array}{c}\text { Controls, } \\
n=12\end{array}$ & \multirow{2}{*}{ Statistic $^{\mathrm{b}}$} & \multirow{2}{*}{$P$-Value } & \multirow{2}{*}{$\begin{array}{c}\text { Cohen } \\
\quad d\end{array}$} \\
\hline & \multicolumn{2}{|c|}{ Mean \pm SD } & & \multicolumn{2}{|c|}{ Mean \pm SD } & & & \\
\hline $\begin{array}{l}\text { Wide Range Achievement Test, } \\
\text { 4th Revision, Reading Subtest }\end{array}$ & $50.3 \pm 5.8$ & $48.4 \pm 6.5$ & .47 & & & & & \\
\hline \multicolumn{9}{|l|}{ Rey Auditory Verbal Learning Test } \\
\hline Initial Learning & $5.6 \pm 2.1$ & $5.9 \pm 1.7$ & .72 & $-0.35 \pm 0.6$ & $-0.03 \pm 0.9$ & -1.02 & .32 & -0.42 \\
\hline Total Learning & $46.5 \pm 10.8$ & $45.2 \pm 11.2$ & .29 & $-0.87 \pm 1.0$ & $0.24 \pm 1.0$ & -2.72 & .01 & -1.11 \\
\hline Delayed Recall & $10.3 \pm 3.5$ & $8.4 \pm 4.4$ & .27 & $-0.60 \pm 1.1$ & $0.04 \pm 1.1$ & -1.42 & .17 & -0.58 \\
\hline \multicolumn{9}{|l|}{ Wechsler Memory Scale_-Revised } \\
\hline \multicolumn{9}{|l|}{ Visual Reproduction } \\
\hline Backward & $7.0 \pm 2.6$ & $6.5 \pm 2.2$ & .61 & $-0.4 \pm 0.9$ & $0.0 \pm 0.9$ & -1.09 & .28 & -0.44 \\
\hline Stroop Color and Word & $-5.0 \pm 9.9$ & $-3.6 \pm 5.3$ & .67 & $-1.0 \pm 1.3$ & $0.0 \pm 1.0$ & -2.11 & .04 & -0.86 \\
\hline & & & & & & & & \\
\hline \multicolumn{9}{|l|}{ Trail-Making Test } \\
\hline Part A (inverse) & $-30.8 \pm 11.9$ & $-30.9 \pm 9.5$ & .97 & $-1.1 \pm 2.1$ & $0.1 \pm 1.1$ & -1.75 & .09 & -0.72 \\
\hline Part B (inverse) & $-115.3 \pm 101.8$ & $-89.75 \pm 41.1$ & .50 & $-0.7 \pm 1.6$ & $0.0 \pm 1.1$ & -1.25 & .22 & -0.51 \\
\hline $\begin{array}{l}\text { Digit Symbol Substitution } \\
\text { Test, total correct }\end{array}$ & $43.3 \pm 13.2$ & $51.3 \pm 13.9$ & .16 & $-0.5 \pm 1.4$ & $0.0 \pm 1.0$ & -1.01 & .32 & -0.41 \\
\hline $\begin{array}{l}\text { Lafayette Grooved Pegboard } \\
\text { Test, mean bilateral } \\
\text { speed (inverse) }\end{array}$ & $-100.5 \pm 35.2$ & $-94.9 \pm 40.2$ & .72 & $-1.0 \pm 2.5$ & $0.0 \pm 1.0$ & -1.28 & .21 & -0.53 \\
\hline Global cognitive change ${ }^{c}$ & & & & $-0.5 \pm 0.5$ & $0.0 \pm 0.3$ & -2.97 & .007 & -1.21 \\
\hline
\end{tabular}

Cognitive tests were part of the Duke Neurologic Outcomes Research Group Neurocognitive Battery. See Appendix 1.

${ }^{a}$ Expressed as reliable change index (RCI) ${ }^{16}$ scores relative to nonsurgical control group test-retest data. See Appendix 1 for RCI formula and methods.

${ }^{\mathrm{b}}$ Independent two-sample $t$-test comparisons (two-tailed).

${ }^{\mathrm{c}}$ Calculated as mean RCI value from all cognitive variables.

\section{White Matter Lesion Volumes}

FLAIR variables of interest were total lesion volume $(\mathrm{mL})$ and the ratio of lesion volume to total intracranial volume (TIV). Independent two-sample tests were conducted between groups for baseline lesion burden and pre- and postoperative changes in total lesion volume and corrected white matter lesion burden $(P<.05$, two-tailed). Baseline TIV-corrected total white matter hyperintensity (WMH) volume and TIV-corrected perioperative white matter legion volume change variables were retained as covariates for entry into the RSFC analyses (see Intrinsic Functional Connectivity section, below) (Appendix S1).

\section{Intrinsic Functional Connectivity}

Intrinsic connectivity contrast (ICC) characterizes the strength of the global connectivity pattern between each gray matter voxel and gray matter voxels in the rest of the brain. In an ICC analysis, values reflect the root sum of squares of the functional connectivity between each gray matter voxel and the remainder of gray matter voxels in the brain $^{18,19}$ (Appendix S1).

Two whole-brain, voxel-wise, random-effects general linear model analyses were conducted to assess for potential differences in the relationship between RSFC of either group and cognition (comparison of ICC by cognition regression slopes). First, any potential differences between groups in the relationship between baseline global cognitive performance and baseline RSFC were examined for, adjusting for age, education, and baseline WMH volume. Then, differences between groups in the relationship between pre- and postoperative RSFC change and global cognitive change (comparison of regression slope differences between groups for ICC change by cognitive change from baseline to 6 weeks) were examined for, adjusting for age, education, and change in perioperative wholebrain WMH lesion volume. Age, education, and WMH volume covariates were mean centered before model insertion. For all analyses, change in RSFC was expressed as 6-week follow-up minus presurgical baseline.

All neuroimaging analyses were conducted using statistical thresholds set to a false discovery rate multiple comparison correction ${ }^{20}$ with a spatial extent $\left(\mathrm{k}_{\mathrm{E}}\right)$ of false discovery rate $P<.01$ and peak voxel $P<.001$.

\section{RESULTS}

\section{Demographic Variables and Cognitive Outcomes}

Presurgical demographic and health variables for participants undergoing cardiac surgery and nonsurgical 
ambulatory controls are noted in Table 1 . No statistically significant differences were found between the groups in age, education, sex, or race. Baseline health factors such as weight, history of diabetes mellitus, and previous history of myocardial infarction also did not differ between groups.

Baseline preoperative intellectual levels were similar between groups (Table 2). No statistically significant baseline differences were found between groups for any of the neurocognitive battery variables (Table 2), although the auditory-verbal list learning abilities $(t=-2.72, P=.01)$ and response inhibition skills $(t=-2.01, P=.04)$ of the surgical group declined after surgery. The mean global RCI score of participants undergoing surgery was significantly lower than that of controls $(t=-2.97, P=.007)$.

\section{White Matter Lesion Volume}

There were no significant differences between participants undergoing surgery and controls in baseline ischemic white matter burden or in changes from baseline to 6 weeks in white matter lesion volume (Table 1). In the surgical group, adjusting for age and education, baseline white matter lesion volume was not significantly associated with overall postoperative cognitive change $(r=-0.48$, $P=.17$ ) or change in any of the other neurocognitive battery variables, with the exception of Trail-Making Test Part A $(r=-0.80, P=.006)$.

\section{Voxel-Wise Intrinsic Functional Connectivity}

There were no significant differences between groups in ICC at baseline or in the relationship between baseline global cognition and baseline ICC, adjusting for age, education, and baseline WMH volume. Two regions of statistically significant positive association between perioperative ICC change and cognitive change exceeded statistical thresholds in the surgical group; neither was detected in the control group. These regions were located in the

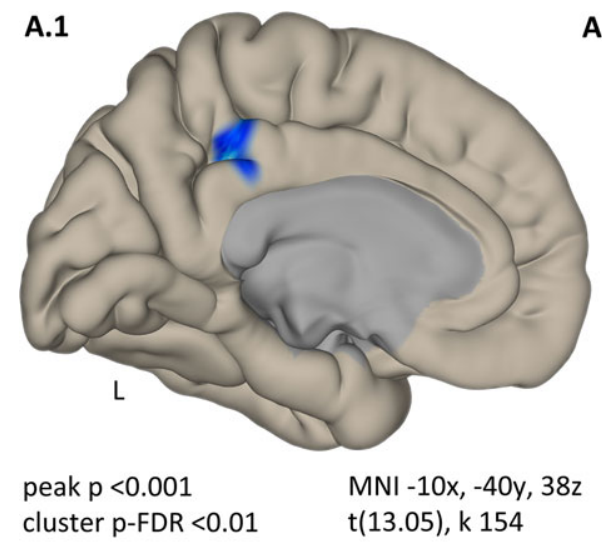

A.2
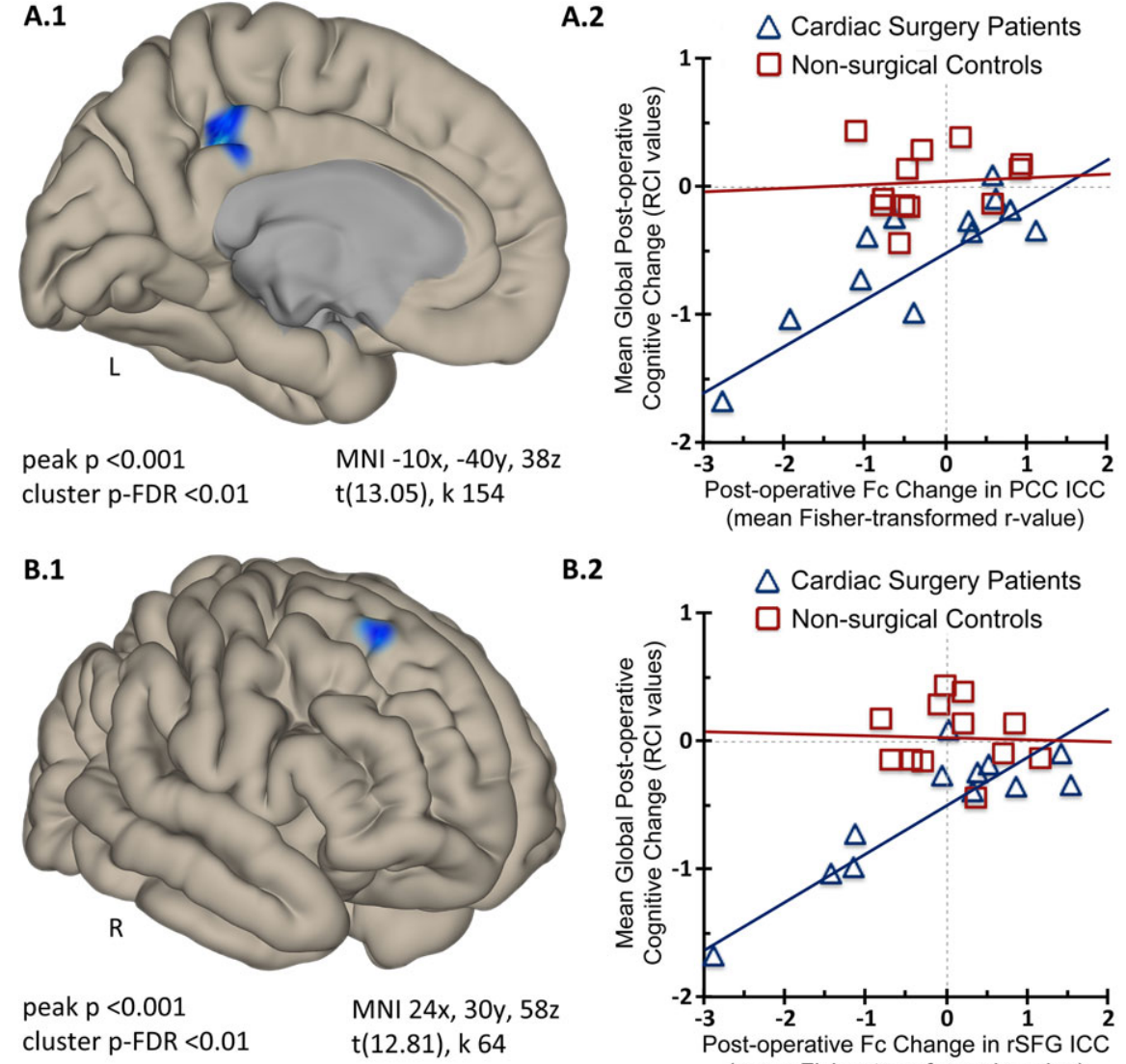

B.2

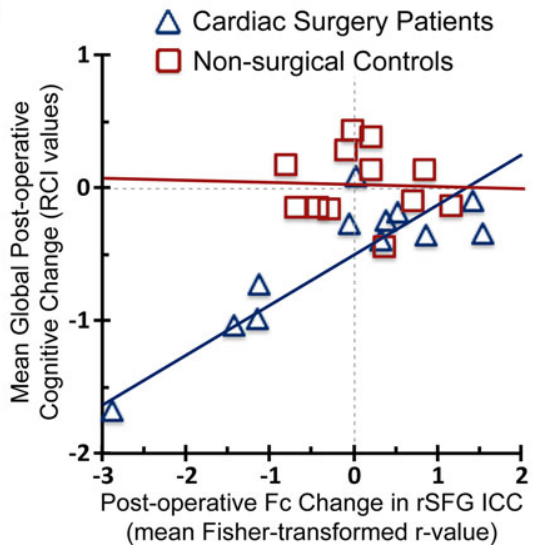

Figure 1. Comparison of regional change in intrinsic functional connectivity associated with postoperative cognitive change between individuals undergoing cardiac surgery and nonsurgical controls. Significant positive relationship between perioperative changes in left posterior cingulate cortex (PCC) intrinsic connectivity contrast (ICC) values and mean global postoperative cognitive change (white region in A.1 and scatterplot A.2) and perioperative changes in right superior frontal gyrus (rSFG) ICC values and mean global postoperative cognitive change (white region in B.1 and scatterplot B.2). Statistical parametric mapping (SPM) random-effects comparison of resting-state functional magnetic resonance imaging voxel-wise intrinsic functional connectivity signal change (ICC) and mean global cognitive change, adjusting for age, education, baseline white matter hyperintensity volume, and perioperative white matter hyperintensity volume change (individuals undergoing surgery $(\mathrm{n}=12)$, controls $(\mathrm{n}=12)$; peak voxel threshold $P<.001$, cluster spatial extent $\left(\mathrm{k}_{\mathrm{E}}\right)$ threshold false discovery rate $\left.P<.01\right)$. Scatterplot $x$-axis $=$ perioperative change in ICC (Fisher-transformed mean correlation coefficients) in the PCC (A.2) and rSFG (B.2); $y$-axis = postoperative change in mean global reliable change index (RCI) values from baseline. See Appendix 1 for greater methodological detail on ICC and RCI values and their calculation. 
posterior division of the left posterior cingulate cortex (PCC), with two local maxima (cluster $154 \mathrm{k}_{\mathrm{E}} ; 13.05 \mathrm{~T}$ $(-10 \mathrm{x},-40 \mathrm{y}, 38 \mathrm{z}), 7.05 \mathrm{~T}(-4 \mathrm{x},-40 \mathrm{y}, 32 \mathrm{z}))$, and the right superior frontal gyrus (SFG), with a single cluster maxima (cluster $64 \mathrm{k}_{\mathrm{E}}$; $12.81 \mathrm{~T}(24 \mathrm{x}, 30 \mathrm{y}, 58 \mathrm{z})$ ) (Figure 1). Fishertransformed correlation coefficients for these regions of significant ICC difference were extracted from the PCC and right SFG regions of interest, averaged within each region of interest ROI, and then individually plotted with the global cognitive change values for participants in the surgical and nonsurgical control groups to allow for visualization of the relationship between FC change and global RCI change. The group-wise differences were observed to reflect a significant positive relationship between postoperative functional connectivity change and global cognition change from baseline to 6 weeks, adjusting for age, education, and cerebral WMH volumes covariates, in the PCC (Figure 1A.2) and right SFG (Figure 1B.2) for participants undergoing surgery that was not seen in the nonsurgical controls.

\section{DISCUSSION}

Postoperative change in global cognitive function is associated with perioperative changes in intrinsic RSFC in DMN-associated PCC and precuneus and right SFG cortical regions (Figure 1). Perioperative RSFC changes in these DMN regions were associated with changes in global cognitive performance, adjusting for age, education, baseline cerebral WMH volume, and perioperative WMH volume change.

Global perioperative volumetric changes in leukoaraiosis did not significantly alter the positive relationship between RSFC change in these DMN regions and global cognitive outcome. Studies have found that perioperative leukoaraiosis volume does not fully account for POCD, ${ }^{21,22}$ although it is unclear whether strategically located perioperative leukoaraiosis, independent of lesion volume, may significantly affect resting-state or task-based functional connectivity in critical global cortical control networks, such as the DMN.

The finding of a continuous relationship between postoperative global cognitive change and RSFC changes in regions of the DMN raises the possibility that POCD and its functional neurological correlates fall along a spectrum rather than being a dichotomous trait. DMN activity is inversely correlated with activity in task-related brain networks such as the dorsal attention network (reviewed $\left.i^{23}\right)$. Cortical association areas, such as the DMN, are known to have greater dendritic spine density than primary unimodal cortices ${ }^{24}$ and higher white matter organization. ${ }^{25}$ The PCC and precuneus region, a central network "hub" of the DMN, ${ }^{26}$ has $40 \%$ higher metabolic activity that most other brain regions ${ }^{27}$ and has been implicated in autobiographical memory to emotional salience, but the exact role of the DMN in cognition is unclear. ${ }^{23}$ The pathophysiological basis for the decrease in DMN "hub" functional connectivity in association with the greater postoperative cognitive decline seen in this sample is unknown. The decrease in intrinsic RSFC in these regions could correlate with or even cause difficulty in shifting between internal processes and external tasks requiring concerted cognitive effort and attention. Altered coordination between latent and active functional brain networks may explain some of the postoperative difficulties in "cognitive efficiency" described by older adults undergoing surgery. ${ }^{28}$ Future studies using resting-state and task-based fMRI studies will be required to address this hypothesis.

In addition to the small sample size, an important limitation of this study is the exclusion of participants with preoperative cognitive impairment (MMSE >27). Thus, it is unclear to what extent these findings may generalize to individuals with baseline cognitive impairment. Understanding the association between postoperative delirium, presurgical cognitive dysfunction, and functional brain connectivity changes that might mediate such associations is important for future studies. Furthermore, these results do not address the root cause of POCD, although they indicate that, in individuals undergoing cardiac surgery without cognitive impairment at presurgical baseline, poorer postoperative global cognitive outcomes are significantly associated with perioperative decreases in intrinsic functional connectivity of regions important to the DMN. These preliminary findings support the need for larger future studies to determine the extent to which varying POCD cognitive and behavioral phenotypes may express altered postoperative resting-state and task-based functional brain connectivity and how pre- and perioperative neurological and psychiatric complications may mediate those relationships.

\section{ACKNOWLEDGMENTS}

The authors would like to acknowledge Rachele Brassard, Yanne Toulgoat-Dubois, Peter Waweru, Erlinda Yeh, Luke Pool, Susan Music, and Natalie Goutkin for their assistance with neurocognitive and neuroimaging data collection and Drs. Allen Song, Director of the Duke Brain Imaging and Analysis Center (BIAC), and Jim Voyvodic for their support and access to BIAC resources, including use of CIGAL software for in-scanner stimulus presentation and physiological data capture.

Research supported in part by National Heart, Lung, and Blood Institute Grants HL109971, HL096978, and HL108280 and National Institute on Aging Grant AG050918.

Conflict of Interest: JNB, TBH, TLB, and JPM derived direct salary support from National Heart, Lung, and Blood Institute Grant HL109971, which partially funded this research effort and manuscript.

Author Contributions: Browndyke: study co-principal investigator, study concept and design, analysis and interpretation of neuroimaging and cognitive data, preparation of manuscript. Berger: neuroanethesiology collaborator, interpretation of data and preparation of manuscript. Harshbarger: biomedical engineering collaborator, analysis and interpretation of neuroimaging data. Smith: behavioral health collaborator, analysis and interpretation of neuroimaging and cognitive data, preparation of manuscript. White: statistical consultant; analysis and interpretation of demographic data; analysis of surgical, neuroimaging, and cognitive data. Bisanar: project coordinator, recruitment and acquisition of subjects and data, preparation of manuscript. Alexander: cardiology collaborator, preparation of manuscript. Gaca: surgical collaborator, acquisition of 
subjects and data. Welsh-Bohmer: neuropsychology collaborator, preparation of manuscript. Newman: anesthesiology collaborator, preparation of manuscript. Mathew: study co-principal investigator, study concept, design and analyses, preparation of manuscript.

Sponsor's Role: Not applicable.

\section{REFERENCES}

1. Berger M, Nadler JW, Browndyke J et al. Postoperative cognitive dysfunction: Minding the gaps in our knowledge of a common postoperative complication in the elderly. Anesthesiol Clin 2015;33:517-550.

2. Terrando N, Monaco C, Ma D et al. Tumor necrosis factor-alpha triggers a cytokine cascade yielding postoperative cognitive decline. Proc Natl Acad Sci U S A 2010;107:20518-20522.

3. $\mathrm{Xu} \mathrm{Z}$, Dong $\mathrm{Y}$, Wang $\mathrm{H}$ et al. Age-dependent postoperative cognitive impairment and Alzheimer-related neuropathology in mice. Sci Rep 2014;4:3766.

4. Tang JX, Mardini F, Janik LS et al. Modulation of murine Alzheimer pathogenesis and behavior by surgery. Ann Surg 2013;257:439-448.

5. Selnes OA, McKhann GM, Borowicz LM Jr et al. Cognitive and neurobehavioral dysfunction after cardiac bypass procedures. Neurolog Clin 2006;24:133-145.

6. Feng X, Degos V, Koch LG et al. Surgery results in exaggerated and persistent cognitive decline in a rat model of the Metabolic Syndrome. Anesthesiology 2013;118:1098-1105.

7. Damoiseaux JS, Rombouts SA, Barkhof $\mathrm{F}$ et al. Consistent resting-state networks across healthy subjects. Proc Natl Acad Sci U S A 2006;103:13848-13853.

8. Raichle ME. The brain's default mode network. Annu Rev Neurosci 2015;38:433-447.

9. van den Heuvel MP, Sporns O. An anatomical substrate for integration among functional networks in human cortex. J Neurosci 2013;33:1448914500 .

10. Sala-Llonch R, Bartres-Faz D, Junque C. Reorganization of brain networks in aging: A review of functional connectivity studies. Front Psychol 2015;6:663.

11. Dennis EL, Thompson PM. Functional brain connectivity using fMRI in aging and Alzheimer's disease. Neuropsychol Rev 2014;24:49-62.

12. Choi SH, Lee H, Chung TS et al. Neural network functional connectivity during and after an episode of delirium. Am J Psychiatry 2012;169:498-507.

13. Inouye SK, van Dyck CH, Alessi CA et al. Clarifying confusion: The Confusion Assessment Method. A new method for detection of delirium. Ann Intern Med 1990;113:941-948.

14. Voyvodic JT, Glover GH, Greve D et al. Automated real-time behavioral and physiological data acquisition and display integrated with stimulus presentation for fMRI. Front Neuroinform 2011;5:27.

15. Behzadi Y, Restom K, Liau J et al. A component based noise correction method (CompCor) for BOLD and perfusion based fMRI. NeuroImage 2007;37:90-101.

16. Duff K. Evidence-based indicators of neuropsychological change in the individual patient: Relevant concepts and methods. Arch Clin Neuropsychol 2012;27:248-261.
17. Silbert B, Evered L, Scott DA et al. Preexisting cognitive impairment is associated with postoperative cognitive dysfunction after hip joint replacement surgery. Anesthesiology 2015;122:1224-1234.

18. Constable RT, Vohr BR, Scheinost D et al. A left cerebellar pathway mediates language in prematurely-born young adults. NeuroImage 2013;64:371-378.

19. Martuzzi R, Ramani R, Qiu M et al. A whole-brain voxel based measure of intrinsic connectivity contrast reveals local changes in tissue connectivity with anesthetic without a priori assumptions on thresholds or regions of interest. NeuroImage 2011;58:1044-1050.

20. Woo CW, Krishnan A, Wager TD. Cluster-extent based thresholding in fMRI analyses: Pitfalls and recommendations. NeuroImage 2014;91:412419.

21. Patel N, Horsfield MA, Banahan C et al. Impact of perioperative infarcts after cardiac surgery. Stroke 2015;46:680-686.

22. Scott DA, Evered LA, Gerraty RP et al. Cognitive dysfunction follows left heart catheterisation but is not related to microembolic count. Int J Cardiol 2014;175:67-71.

23. Leech R, Sharp DJ. The role of the posterior cingulate cortex in cognition and disease. Brain 2014;137:12-32.

24. Jacobs B, Schall M, Prather M et al. Regional dendritic and spine variation in human cerebral cortex: A quantitative Golgi study. Cereb Cortex 2001;11:558-571.

25. Collin G, Sporns O, Mandl RC et al. Structural and functional aspects relating to cost and benefit of rich club organization in the human cerebral cortex. Cereb Cortex 2014;24:2258-2267.

26. Achard S, Salvador R, Whitcher B et al. A resilient, low-frequency, smallworld human brain functional network with highly connected association cortical hubs. J Neurosci 2006;26:63-72.

27. Raichle ME, MacLeod AM, Snyder AZ et al. A default mode of brain function. Proc Natl Acad Sci U S A 2001;98:676-682.

28. Schwarz N, Kastaun S, Schoenburg M et al. Subjective impairment after cardiac surgeries: The relevance of postoperative cognitive decline in daily living. Eur J Cardiothorac Surg 2013;43:e162-e166.

\section{SUPPORTING INFORMATION}

Additional Supporting Information may be found in the online version of this article:

Appendix S1. Detailed study participant information, perioperative management, neurocognitive assessment procedures, characterization and measurement of cognitive change, postoperative delirium assessment procedures, neuroimaging procedures, and data acquisition and analyses.

Please note: Wiley-Blackwell is not responsible for the content, accuracy, errors, or functionality of any supporting materials supplied by the authors. Any queries (other than missing material) should be directed to the corresponding author for the article. 